\title{
Interfacial and Wetting Properties of Poly(3-hexylthiophene)-water systems
}

\author{
Yeneneh Y. Yimer ${ }^{\mathrm{a}, \mathrm{d}}$, Brandon Yang ${ }^{\mathrm{b}, \mathrm{e}}$, Ram S. Bhatta ${ }^{\mathrm{a}}$, Mesfin Tsige $^{\mathrm{a}, \mathrm{c}}$ \\ ${ }^{a}$ Department of Polymer Science, The University of Akron, Akron, OH 44325 \\ ${ }^{b}$ The Harker School, San Jose, CA, 95130 \\ ${ }^{c}$ Corresponding author: mtsige@uakron.edu \\ ${ }^{d}$ Currently: Department of Chemical Engineering,University of Washington, Seattle, WA 98195 \\ ${ }^{e}$ Currently: Undergraduate Student at Stanford University, Stanford, CA 94305
}

\begin{abstract}
The wetting behavior of water on different types of poly(3-hexylthiophene)(P3HT) surfaces is studied using molecular dynamics simulations. All the different P3HT surfaces are found to be hydrophobic with water contact angles greater than $90^{\circ}$. The water contact angle on hexyl exposed crystalline $\mathrm{P} 3 \mathrm{HT}$ films is about $20^{\circ}$ larger than its contact angle on backbone-exposed crystalline P3HT films. However, all the films show comparable interfacial surface energies at the $\mathrm{P} 3 \mathrm{HT} /$ water interface. The simulation results are in very good agreement with experimentally measured water contact angles on P3HT films and also interfacial tensions.
\end{abstract}

Keywords: Molecular Dynamics Simulation, Conducting Polymer, Wetting, Interfacial Properties and Contact Angle

\section{Introduction}

Semiconducting polymers have great potential applications in electronic and optoelectronic devices such as photovoltaics, light-emitting diodes and field-effect transistors.[1] For the past few decades, these materials have received significant attention due to their solution processability and unique combination of mechanical flexibility and excellent photophysical properties. Among semiconducting polymers, poly(3-hexylthiophene) (P3HT) is widely studied due to its high conductivity, thermal stability, and compatibility with wet processing techniques.[2] Currently, the two most important areas of polymer solar cell research are in improving device efficiency and in enhancing stability. To improve solar cell device efficiency and lifetime, it is important to understand the mechanisms of device degradation caused by physical and chemical changes.[3] Physical degradation occurs mainly due to changes in structure and morphology over time. Microstructures formed during device construction are often not thermodynamically stable, and microstructural changes over time lead to reductions in solar cell device performance. Chemical degradation of organic solar cells is mainly due to the reaction of oxygen and water with the electrodes and the active polymer layer. Oxygen and water can be introduced during device fabrication but are more deleterious over the solar cell's lifetime when they diffuse into finished devices.

Moreover, conductive polymers have also been used for biomedical applications such as biosensors and neural probes and in tissue engineering.[4] Of the wider class of conducting 
polymers, thiophene-based devices have shown significant promise. Hydrophobicity and lack of cytotoxicity make thiophene-based polymers useful as synthetic cell scaffolds[5], and by varying the lengths of alkyl side chains, surface properties can be tuned for selective adsorption of proteins, [6] and to design reversible superhydrophilic-to-superoleophobic surfaces.[7] The wettability of the surface is directly related to the hydrophobicity/hydrophilicity of the surface.

In order to create more efficient solar cells and biomedical devices, understanding the interfacial properties of thiophene-based polymers such as $\mathrm{P} 3 \mathrm{HT}$ at polymer/water interfaces is of major importance. In this study, we are mainly interested in understanding the wetting of P3HT by water. The interactions of different parts of the P3HT chains (thiophene rings and hexyl side chains) at the water interface determine the wetting behavior. To better understand the wetting properties of $\mathrm{P} 3 \mathrm{HT}$, knowledge of the structure and ordering of the molecular groups at the interface is vital. Molecular dynamics (MD) simulation is one of the most commonly used techniques to determine such quantities at the atomic level. MD simulations rely on force field parameters to accurately describe the physical properties of a given system. In our recent work, we have developed ab initio-based optimized force field parameters for P3HT.[8] These force field parameters are used in the present study to investigate the wetting behaviour of water on three different types of P3HT surfaces: amorphous and two different types of crystalline P3HT surfaces where in the latter case the P3HT chains at the surface are in either edge-on orientation (only hexyl side chains are pointing normal and facing the surface) or face-on (thiophene rings and hexyl side chains are in the plane of the surface).

The present study primarily has two aims: (1) to characterize the wetting behavior and interfacial properites of $\mathrm{P} 3 \mathrm{HT} /$ water systems and (2) to validate the applicability of the new force field parameters of $\mathrm{P} 3 \mathrm{HT}[8]$ to interfacial properties. More specifically, we have investigated the effect of P3HT surface morphology and chain length on the wetting behaviour of water on P3HT surfaces at $300 \mathrm{~K}$. In order to have a molecular level information of the wetting properties, we have calculated contact angles, molecular orientations and density profiles (mass and number) of the P3HT/water system in the normal to the interface direction. In Section 2, we discuss the details of our simulation procedures. Calculations of contact angle, interfacial energy, mass and number density profiles and orientational ordering and the discussion of those results, are presented in Section 3. A summary of our findings and the conclusions drawn are presented in Section 4.

\section{Methodology and Simulation Details}

In this study, we have employed all-atom molecular dynamics simulations using our recent force field parameters for P3HT that are reported in our recent study.[8] This force field was shown to reproduce satisfactorily several physical quantities, such as, crystalline structure, mass density, melting temperature, glass transition temperature and surface tension of P3HT. The force field is based on the OPLS-AA form, specifying the total potential energy as the sum of bonding energies (bond-stretching, angle-bending and torsion) and nonbonding energies (Coulomb and van der Waals).

Water was modeled using extended simple point charge (SPC/E) model[9] which is one of the most commonly used water models for studying the physical properties of bulk water as well as water near surfaces. The O-H bond distances and $\mathrm{H}-\mathrm{O}-\mathrm{H}$ bond angles were constrained using the SHAKE algorithm.[10] Van der Waals interactions (for both P3HT and water) were defined by the Lennard-Jones (LJ) potential, $U_{i j}^{L J}=4 \epsilon_{i j}\left[\left(\sigma_{i j} / r_{i j}\right)^{12}-\left(\sigma_{i j} / r_{i j}\right)^{6}\right]$, where $\sigma_{i j}$ and $\epsilon_{i j}$ are the $\mathrm{LJ}$ diameter and interaction strength, respectively. The LJ parameters $\left(\sigma_{i j}\right.$ and $\left.\epsilon_{i j}\right)$ for 
interactions between different atomic species (for both P3HT and water) were calculated using geometric mixing rules $\left(\epsilon_{i j}=\sqrt{\epsilon_{i} \epsilon_{j}}\right.$ and $\left.\sigma_{i j}=\sqrt{\sigma_{i} \sigma_{j}}\right)$.

In order to investigate the effect of molecular weight on the wetting behavior of water on P3HT surfaces, two different films with 100\% regioregular P3HT chains but with different chain lengths were prepared for each of the three different types of P3HT surfaces. One of the films has 64 20-monomer P3HT chains (total system size: 32,000 atoms) while the other has 8040 monomer chains (total system size: 80,000 atoms). Initially, the crystalline P3HT systems were generated by placing P3HT chains in a simulation box such that the backbone of the chains were oriented along the $x$-axis with $\pi-\pi$ stacking along the $y$-axis and hexyl side chains oriented along the $z$-axis. The initial box dimensions were $80 \AA \times 65 \AA \times 69 \AA$ and $158 \AA \times 100 \AA \times 69 \AA$ for systems with 20 -monomer chains and 40 -monomer chains, respectively. To minimize the effect of sudden temperature change on the systems morphology both systems were heated from $20 \mathrm{~K}$ to $300 \mathrm{~K}$ in increments of $20 \mathrm{~K} / \mathrm{ns}$ in an NPT ensemble and equilibrated for an additional $5 \mathrm{~ns}$ at $300 \mathrm{~K}$. From each of these systems, two free-standing P3HT films were generated by exposing either the hexyl side chains (edge-on orientation) or the chain backbones (face-on orientation) of the $\mathrm{P} 3 \mathrm{HT}$ chains to vacuum. By removing periodicity along the $z$-axis, the films with hexyl side chains exposed to vacuum were generated. The films with backbones exposed to vacuum were generated in two steps: first, periodicity along the $y$-axis was removed and then the film was rotated by $90^{\circ}$ about the $x$ - axis so that the normal of the film surface to be along the $z$-axis. To generate amorphous films, the crystalline P3HT bulk systems were further heated to $600 \mathrm{~K}$ in increments of $20 \mathrm{~K} / \mathrm{ns}$ in the NPT ensemble and then equilibrated at $600 \mathrm{~K}$ for an additional $5 \mathrm{~ns}$. The amorphous films were generated by removing the periodicity along the $z$-axis, then cooling the film from $600 \mathrm{~K}$ to $300 \mathrm{~K}$ in decrements of $10 \mathrm{~K} / \mathrm{ns}$ followed by an additional equilibration of $5 \mathrm{~ns}$ at $300 \mathrm{~K}$.

To generate water slabs, first a large bulk water system was equilibrated at $300 \mathrm{~K}$ in an NPT ensemble. For each P3HT film, two water slabs were cut out of this equilibrated bulk water system for contact angle calculations and for orientation calculations as described below. The water slab dimensions for the contact angle calculations were $45 \AA \times L_{y} \times 45 \AA$, where $L_{y}$ is the box dimension of a P3HT film along the $y$-axis. The water takes on a cylindrical geometry once equilibrated on the P3HT surface, as shown in Figure 1. This cylindrical geometry reduces the effect of finite size on the contact angle, unlike hemispherical droplets, by eliminating the influence of the three-phase line tension.[11,12,13] For orientation calculations, the water slabs had the same surface area as the P3HT film and were initially placed at a distance of $2.5 \AA$ above the surface of the P3HT film. To ensure periodic images did not interact with the interface, the simulation box size was increased by $100 \AA$ along the $z$-axis (normal to the interface). The $\mathrm{P} 3 \mathrm{HT} /$ water systems were then run in the NVT ensemble at $300 \mathrm{~K}$ for at least $5 \mathrm{~ns}$; with frequent dumping in the last $1 \mathrm{~ns}$ of the simulation, the positions of the atoms were dumped every $1 \mathrm{ps}$ and 1000 different trajectories were used for analysis.

All simulations were performed using the Large-scale Atomic/Molecular Massively Parallel Simulator (LAMMPS) package[14] and the velocity-Verlet algorithm was used for integration of the equations of motion with a time step of $1 \mathrm{fs}$. To maintain the temperature of the system in both NVT and NPT ensembles, a Nosé-Hoover thermostat with a damping time of $100 \mathrm{fs}$ was used. To keep the pressure of the system at $1 \mathrm{~atm}$ in the NPT ensemble, a Nosé-Hoover barostat with $1 \mathrm{ps}$ damping time was also used. For nonbonded interactions, a cut-off distance of $12 \AA$ was used; a long-range van der Waals tail correction was added to the energy. The long-range Coulomb interactions (beyond the cutoff distance) were calculated using the particle-particle/particle-mesh (PPPM) Ewald algorithm.[15] 


\section{Results and Discussions}

\subsection{Contact Angle}

The wetting behaviour of a material surface by a liquid is mainly determined by the chemistry of the surface (interactions), the surface structure and the dynamics of the liquid/film interface. The degree of wetting of a surface is commonly characterized by measuring the contact angle of the liquid droplet. A contact angle is a macroscopic parameter which is usually determined from the shape of the equilibrated droplet in both experiments and simulations. On a P3HT film surface the contact angle is thus obtained from the equilibrium shape of the water droplet on the surface. It is assumed that the water droplet has its equilibrium shape when the distance between the center of mass of the P3HT film and the center of mass of the water droplet remains constant with time. Representative $\mathrm{P} 3 \mathrm{HT} /$ water snapshots for the initial and equilibrated configurations are shown in Figure 1.
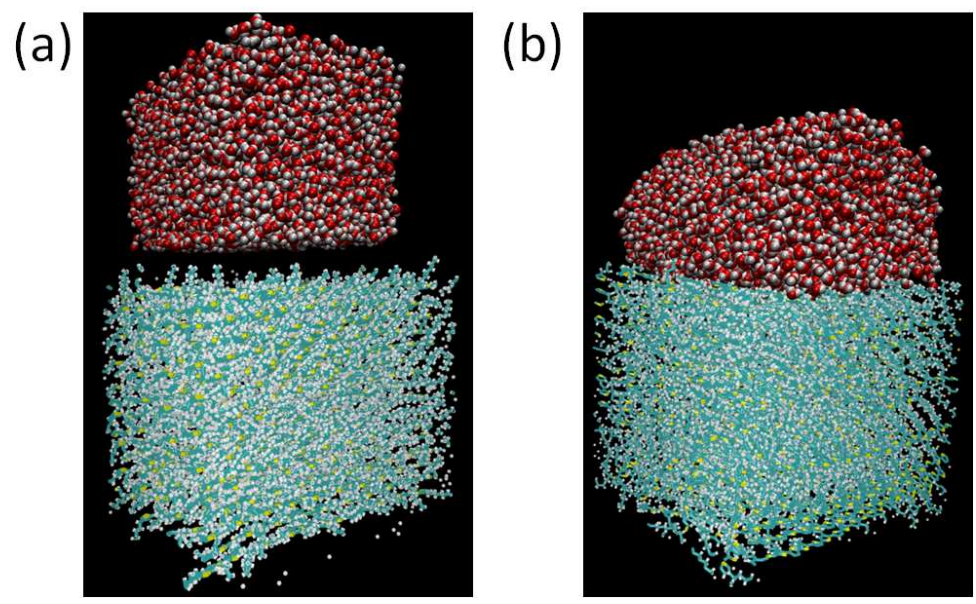

Figure 1: Snapshots of backbone-exposed 20-monomer P3HT/water systems, (a) initial configuration and (b) equilibrated configuration. Note that periodic boundary conditions in the directions parallel to the interface have been applied.

In order to calculate the contact angle of the water droplet, we determined its equilibrium mass density profile. The mass density at a given value of $(x, z)$ was calculated by partitioning the water droplet into rectangular cells of size $0.5 \AA \times L_{y} \times 0.5 \AA$ and dividing the total mass of the water molecules in each cell by the cell volume. Figure 2 shows the mass density profiles of water droplets on six different P3HT surfaces. From the density profiles, the Gibbs dividing surface for the water/vapor interface was determined to be the locus of points for which the mass density of water is half of its bulk density $\left(1.0 \mathrm{~g} / \mathrm{cm}^{3}\right)$. To approximate the Gibbs dividing surface, a circle was fitted to those data points, as shown in Figure 3. The choice of the horizontal reference line for calculation of the contact angle was determined to be the location at which the density of the water droplet near the interface has fully recovered to its bulk density. The contact angle was measured by calculating the angle between this horizontal line and the tangent line to the fitted circle at the intersection point as shown in Figure 3. Moving the location of the horizontal line by a few angstroms towards the Gibbs dividing surface at the water/P3HT interface resulted in an over all increase of the contact angle by up to 3 degrees. The main source of error in the contact 

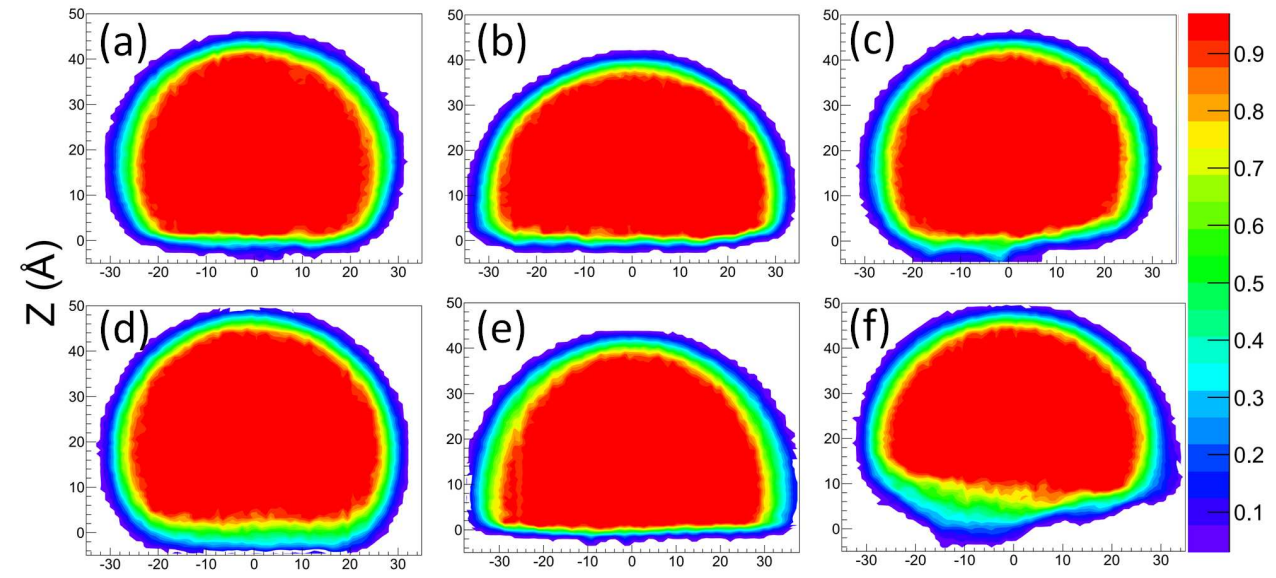

Figure 2: Equilibrated density profiles of water droplets showing the wetting behavior of different P3HT surfaces. Top row and bottom row are for films containing 20-monomer and 40-monomer chains, respectively, and from left to right: hexyl-exposed ( $a$ and d), backbone-exposed (b and e) and amorphous (c and f). The colors represent mass density in $\mathrm{g} / \mathrm{cm}^{3}$ as shown in the key on the right.

angle calculation is in the determination of the location of the horizontal line, and as such, we report a maximum error of $\pm 3^{\circ}$ for the reported contact angles.

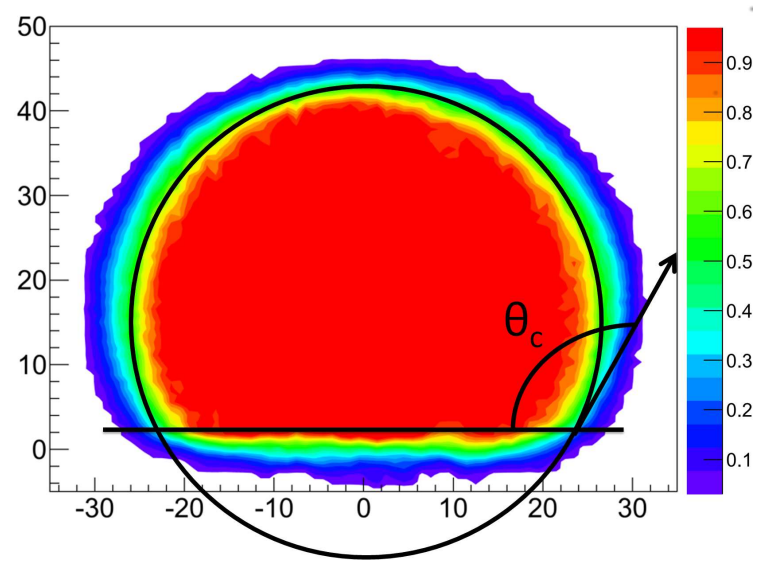

Figure 3: Density profile for 20-monomer P3HT hexyl-exposed films. The circle is a fit to the water/vacuum Gibbs dividing surface. The contact angle $\left(\theta_{c}\right)$ was determined by calculating the angle between the line tangent to the water/vapor interface and a horizontal line parallel to the $\mathrm{P} 3 \mathrm{HT} /$ water interface.

To determine the time evolution of the equilibrium water shape, we calculated the contact angle of the water droplet as a function of time. The contact angle and the corresponding snapshot of the water droplet for the 20-monomer hexyl-exposed P3HT film system case can be seen in Figure 4. Here, one can see that the contact angle increases with increasing time and the droplet assumes its equilibrium shape within 600 ps. Hence, simulations of $2 \mathrm{~ns}$ in length should be 
sufficient to obtain an equilibrium droplet shape. We extended our runs to 5 ns to make sure that the surface structure of the P3HT film did not show any noticeable structural change for an extended period of time.

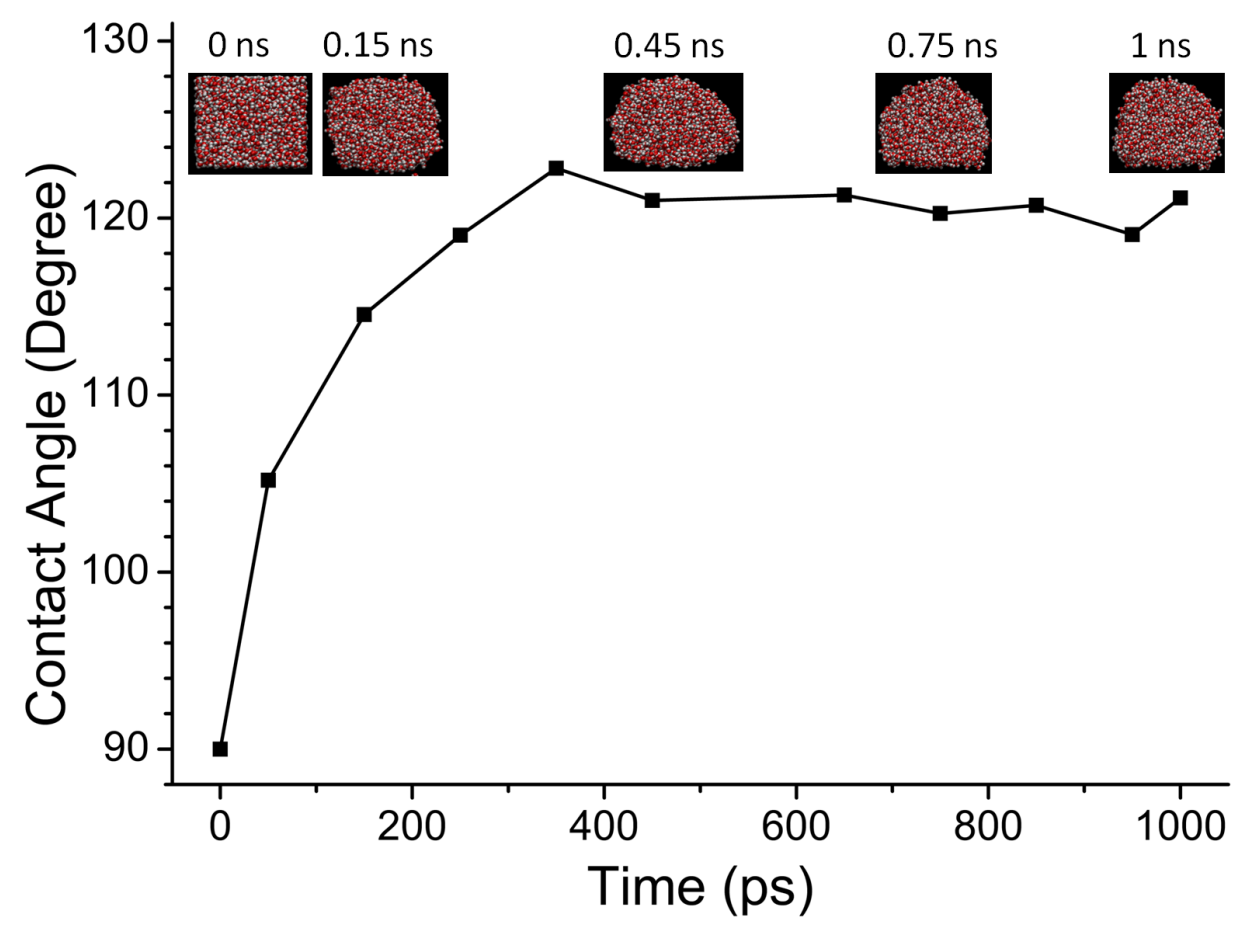

Figure 4: Time evolution of water contact angle on 20-monomer P3HT hexyl-exposed surface. The corresponding snapshots of water molecules are shown along the top.

The calculated water contact angles on different P3HT surfaces are given in Table 1. As can be seen, all contact angles are greater than $90^{\circ}$, confirming that all the P3HT surfaces are hydrophobic. The water contact angles on the amorphous films are much closer to the contact angles on the hexyl exposed films than on the backbone-exposed films. This is in agreement with our recent result $[16,17]$ where we showed for a free-standing amorphous P3HT film that the hexyl side chains strongly dominate at the surface and orient normal to the surface as we get closer to its glass transition temperature suggesting that the films surface property should be mainly determined by the hexyl side chains on the surface.

Table 1: Water contact angle on different P3HT film surfaces

\begin{tabular}{ccc}
\hline \hline & 20-monomer & 40-monomer \\
\hline Hexyl & 122 & 120 \\
Backbone & 103 & 100 \\
Amorphous & 117 & 112 \\
\hline
\end{tabular}


For crystalline P3HT films, the difference in contact angles between 40-monomer and 20monomer cases is about $\sim 2^{\circ}$, which is within the error of our calculations. However, for the amorphous films this difference is about $\sim 5^{\circ}$. The large difference for the amorphous films is likely due to the difference in surface roughness of the films, which can also be observed from the water density profiles in Figure 2. The surface roughness values for the amorphous 20and 40-monomer films are $2.56 \AA$ and $4.83 \AA$, respectively. Experimentally measured contact angle values are in the range of $102^{\circ}$ to $109^{\circ},[18,19,20,21]$ in good agreement with our simulation results (backbone-exposed and amorphous films). The variation in the experimentally measured contact angles is due to differences in surface morphology as a result of different film processing conditions. The actual P3HT films are semicrystalline with both hexyl and backbone groups present at interfaces. However, our model system is either pure crystalline (hexyl- or backbone-exposed) or amorphous, and thus predicts an upper (hexyl-exposed) and lower bounds (backbone-exposed) for the contact angle values.

In a recent MD simulation study, Vanzo et al.[13] investigated the effect of the alkyl chain length on surface wettability of alkyl-functionalized graphene. They found that water contact angle increases with increasing alkyl chain length, and in butylated form, the contact angle saturates at $114^{\circ}$. The saturation in the contact angle was attributed to the inability of water molecules to penetrate into the $n$-alkyl molecular brush and interact with carbon atoms of the underlying lattice.[13] Their contact angle is in good agreement with the contact angle we calculated for hexyl-exposed films. The small difference in contact angle between our calculations and Vanzo et al.[13] may be due to the differences in the number of $n$-alkyl groups per surface area (4.01 $\mathrm{nm}^{-2}$ in Vanzo vs. $3.44 \mathrm{~nm}^{-2}$ in this work).

Table 2: Interfacial energy at P3HT/vacuum[8] and at P3HT/water interfaces

\begin{tabular}{|c|cc|cc|}
\hline \hline & \multicolumn{2}{|c|}{ 20-monomer } & \multicolumn{2}{c|}{ 40-monomer } \\
\hline & $\gamma_{S V}(\mathrm{mN} / \mathrm{m})$ & $\gamma_{S L}(\mathrm{mN} / \mathrm{m})$ & $\gamma_{S V}(\mathrm{mN} / \mathrm{m})$ & $\gamma_{S L}(\mathrm{mN} / \mathrm{m})$ \\
\hline Hexyl & 30.4 & 64.1 & 29.7 & 61.5 \\
Backbone & 57.4 & 71.7 & 54.7 & 65.7 \\
\hline
\end{tabular}

The P3HT/water (solid/liquid) interfacial energy can be calculated using the equilibrium contact angle determined above. The contact angle arises from a thermodynamic equilibrium between the three phases, related by Young's equation[22]

$$
\gamma_{S V}=\gamma_{S L}+\gamma_{L V} \cos \left(\theta_{c}\right)
$$

where $\gamma_{S V}, \gamma_{S L}$ and $\gamma_{L V}$ represent the solid/vapor, solid/liquid, and liquid/vapor interfacial energies, respectively, and $\theta_{c}$ denotes the equilibrium contact angle. At $300 \mathrm{~K}, \gamma_{L V}$ for the SPC/E water model is $63.6 \mathrm{mN} / \mathrm{m}[23]$ and the calculated interfacial energies at the P3HT/water interface $\left(\gamma_{S L}\right)$ using equation (1) are provided in Table $2-$ the $\gamma_{S V}$ values for P3HT films at $300 \mathrm{~K}$ are taken from our previous study[8] and are also included in the Table. For backbone-exposed $\mathrm{P} 3 \mathrm{HT}$, the $\gamma_{S V}$ is about $26 \mathrm{mN} / \mathrm{m}$ larger and contact angle is about $19.5^{\circ}$ smaller than for hexylexposed P3HT. However, the difference in $\gamma_{S L}$ values between backbone- and hexyl-exposed surfaces are $7.6 \mathrm{mN} / \mathrm{m}$ and $5.2 \mathrm{mN} / \mathrm{m}$ for 20 - and 40 -monomer films, respectively. As the contact angle results implied and also further verified in the next sub-section, the amorphous films are expected to have a similar interfacial energy $\left(\gamma_{S L}\right)$ as the hexyl-exposed films and, as such, no attempt was made to determine their $\gamma_{S V}$ values that are needed for calculating $\gamma_{S L r}$ in equation (1). 


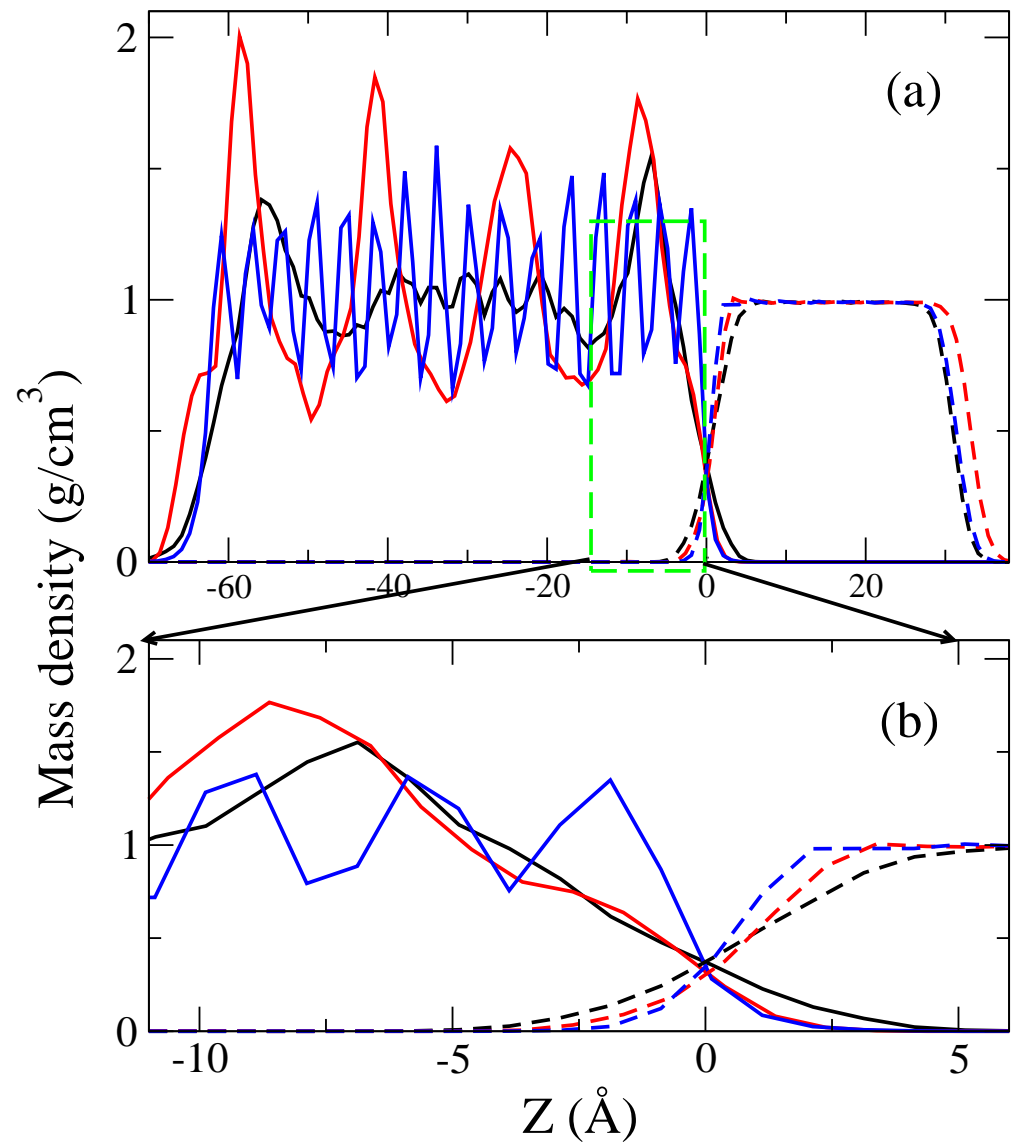

Figure 5: Mass density profile along the $z$-axis for hexyl-exposed (solid red line), backbone-exposed (blue solid line) and amorphous (black solid line) films are shown in the top figure for the 20-monomer case. The bottom figure is a magnification of the P3HT/water interface shown by the green rectangle in the top figure. The corresponding density profiles for water are shown in broken lines. 


\subsection{Molecular Orientation}

The ordering of molecules at interfaces can be described by looking at the density (mass and number) and orientation of molecules along the normal to the surface direction. The mass density profile across the interface can give us a first hand approximation and insight of the ordering of the molecules at the interface. The mass density profile $\rho(z)$ was calculated separately for water and P3HT by partitioning the simulation box into $1 \AA$ A-thick bins along the $z$-axis and calculating the total mass of atoms in each bin per partition volume. The mass density profiles for the three 20-monomer P3HT film/water slab cases (hexyl-exposed, backbone-exposed and amorphous) are shown in Figure 5. As can be seen, the interfacial width for water slab on amorphous film is slightly larger than for water slab on backbone- and hexyl-exposed films. This is mainly due to the difference in surface roughness; the calculated surface roughnesses for amorphous, hexyl-exposed and backbone-exposed films are $2.56 \AA, 1.88 \AA$ and $1.96 \AA$, respectively. The density profiles for both hexyl- and backbone-exposed P3HT films show ordering of the chains corresponding to their crystalline packing.

A closer look of the P3HT density profile for each of the cases at the P3HT/water (Figure 5(b)) and P3HT/vacuum interfaces reveals that there are no observable differences in the density profile at the two interfaces, and this indicates that no significant structural changes were induced in the P3HT film due to the presence of water. Furthermore, the feature of the P3HT density profiles of hexyl-exposed and amorphous P3HT films are similar at the two interfaces which was expected on the basis of the comparable contact angle values of the two surfaces discussed above. In addition to the crystalline films, the amorphous film shows strong ordering at the two interfaces, as indicated by the peak in the mass density profile, but not in the middle region of the film (bulk). Such ordering at interfaces can be better explained by calculating the number density and orientation of the different groups of the P3HT molecules in the films.

To calculate molecular orientation and number density, P3HT monomers were subdivided into three representative groups; thiophene rings, hexyl groups and methyl groups. The locations of methyl and hexyl groups were defined by the geometric center of the atoms in the respective groups, whereas the locations of thiophene rings and water molecules were represented by the locations of the sulfur atoms and oxygen atoms in those groups, respectively. A bin size of $1 \AA$ was used in molecular orientation and number density calculations, same bin size as for the mass density calculation above. The number density at a given value of $z$ was calculated by summing over the number of target groups in each bin and dividing by partition volume. The molecular orientations of water molecules and different groups in P3HT were determined by calculating the average cosine of the angle between the orientation vector and the $z$-axis (i.e., the surface normal) as a function of $z$. The orientational vector is defined as follows: water molecules vector originating from the oxygen atom and ending at the midpoint of the line segment joining the hydrogen atoms; thiophene rings - the vector originating from the thiophene sulfur atom and ending at the carbon atom at the ring 3-position (see Figure 6(a)); hexyl groups - vector originating from the carbon atom at the ring 3-position and ending at the carbon atom of the methyl group (see Figure 6(b)); methyl groups - vector originating from the carbon atom of the methyl group and ending at the geometric center of the methyl hydrogens (see Figure 6(c)).

For water slabs equilibrated on 20-monomer P3HT film cases, the number density and orientation of water molecules and the three groups of the P3HT chains are shown in Figure 7. As can be seen from the number density profiles, for hexyl-exposed and amorphous P3HT film cases, the hexyl and methyl groups enrich the interfaces. However, for backbone-exposed films, all three groups of the $\mathrm{P} 3 \mathrm{HT}$ chains are present at the interface with equal proportions as expected since 


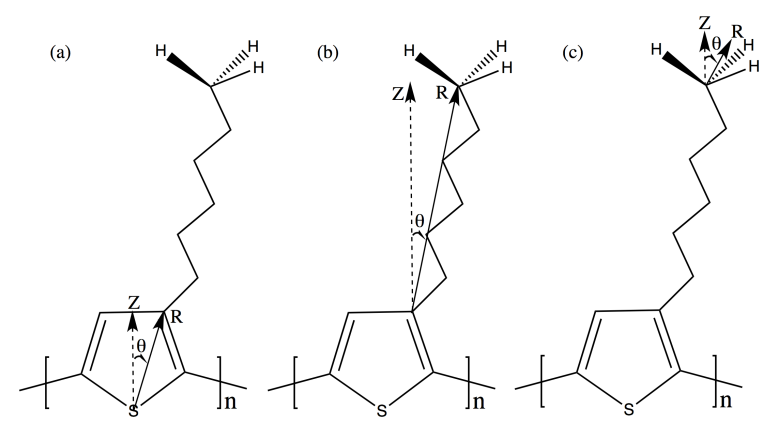

Figure 6: Definition of the orientational angle $\theta$ of different parts of P3HT chains: (a) thiophene ring, (b) hexyl group, and (c) methyl group.

no significant structure changes were observed at the interfaces. In all P3HT films, the three groups of the $\mathrm{P} 3 \mathrm{HT}$ chains at the interfaces (with vacuum and water) are preferentially oriented toward the surface normal. For amorphous P3HT films, to minimize the interfacial energies, the hexyl side chains (hexyl and methyl groups) come to the surface. This leads to ordering of thiophene rings at interfaces which contributes to the observed peaks in mass density profile shown in Figure 5.

Similar orientations were observed for water molecules at the interfaces with hexyl-exposed and amorphous films, as shown in Figure 7. In both P3HT films, the surfaces are dominated by the hexyl and methyl groups and to minimize the interfacial energy, the water molecules order and orient by exposing the hydrogen atoms to the P3HT side; similar water molecule orientation is also observed at the water/vapor interface. Even though the hexyl-exposed surface is smoother compared to the amorphous surface, the contact angles on the two surfaces are found to be comparable. Thus, the surface chemistry is the dominating factor rather than the small difference in surface roughness between the two systems. For the backbone-exposed films the water molecules at the P3HT/water interface seem to show no strong preferred orientational ordering. This is a result of the presence of all three groups of the P3HT chain at the interface. The hexyl and methyl groups are hydrophobic, thus the water molecules expose their hydrogen atoms to these groups at the interface. The thiophene rings, however, are polar (less hydrophobic) thus the water molecules expose their oxygen atoms towards the thiophene rings at the interface. Adding these two opposing orientations of the water molecules at the P3HT/water interface cancels out and that is what is reflected in the orientation profile in Figure 7(b). A similar behavior (not shown) in both number density and orientation of the three groups of the P3HT chains and water molecules was observed for the 40-monomer P3HT/water systems .

\section{Conclusions}

Using all-atom molecular dynamics simulations, we have modeled the wetting behaviour of water on different P3HT surfaces at $300 \mathrm{~K}$. In this study, we have - for the first time - employed a force field specifically optimized for investigating interfacial properties of P3HT. In our simulations, six different types of P3HT films were considered - films with two different molecular weights in three different types of molecular ordering of the P3HT molecules at the interfaces (hexyl-exposed (edge-on), backbone-exposed (face-on), and amorphous). To characterize the 

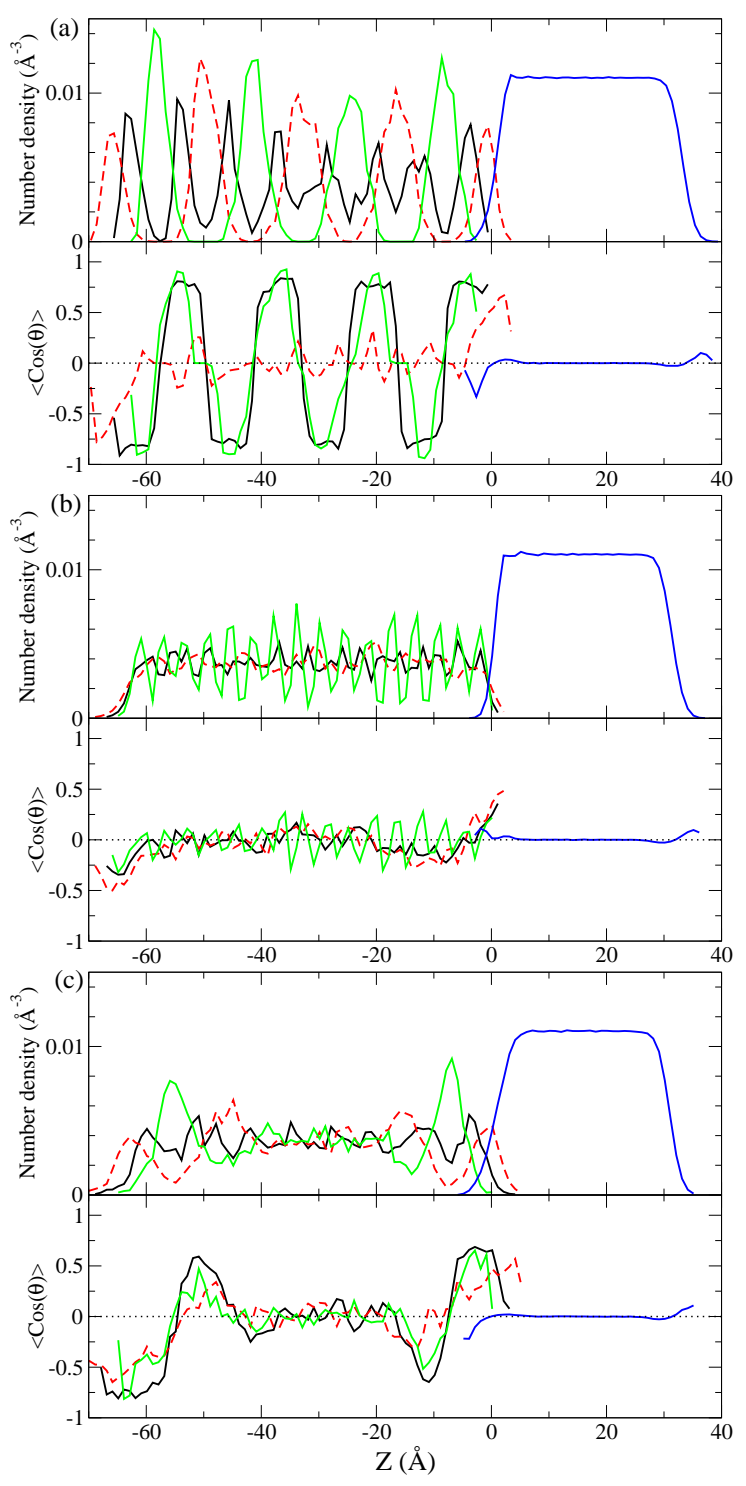

Figure 7: Number density and orientation profiles for three different 20-monomer P3HT films - hexyl-exposed (a), backbone-exposed (b) and amorphous (c) - are shown. In the figure, the profiles for water molecules are shown in solid blue lines, thiophene rings are shown in solid green lines, hexyl groups are shown in solid black lines, and methyl groups are shown in broken red lines. Number density for water molecules was scaled by a factor $1 / 3$, in order to plot it on same scale with different groups of the P3HT molecules. 
interfacial properties of the $\mathrm{P} 3 \mathrm{HT} /$ water systems, we calculated water contact angles, interfacial energies, and molecular orientations. All contact angle values were greater than $90^{\circ}$, as expected for hydrophobic P3HT films. The contact angles for hexyl-exposed films were greater than for backbone-exposed films by about $20^{\circ}$. The contact angles did not show strong dependence on P3HT molecular weight. The amorphous P3HT films showed similar surface properties as the hexyl-exposed films. The calculated contact angles are in agreement with experimentally measured values.[18, 19, 20, 21] Though large differences in surface tension values (P3HT/vacuum) and contact angle values were observed between hexyl- and backbone-exposed films, their interfacial surface tension with water was found to be comparable. The water molecules' orientations on hexyl-exposed and amorphous films were similar because hexyl side chains enriched both surfaces. Water molecules on backbone-exposed films, however, did not show noticeable preferred orientation due to competition in the interactions between water with hexyl side chains and water with thiophene rings. The thiophene rings of the amorphous $\mathrm{P} 3 \mathrm{HT}$ films showed ordering at both $\mathrm{P} 3 \mathrm{HT} /$ vacuum and P3HT/water interfaces.

Acknowledgements The authors are grateful to the National Science Foundation (Grant No. DMR0847580) for financial support.

\section{References}

[1] B. Schwartz, Annual Review of Physicsal Chemistry 54 (2003) 141 - 172.

[2] Z. Bao, A. Dodabalapur, A. J. Lovinger, Applied Physics Letters 69 (1996) $4108-4110$.

[3] M. Jørgensen, K. Norrman, F. C. Krebs, Solar Energy Materials and Solar Cells 92 (2008) 686 - 714.

[4] N. K. Guimard, N. Gomez, C. E. Schmidt, Progress in Polymer Science 32 (2007) 876 - 921.

[5] A. F. Quigley, K. Wagner, M. Kita, K. J. Gilmore, M. J. Higgins, R. D. Breukers, S. E. Moulton, G. M. Clark, A. J. Penington, G. G. Wallace, D. L. Officer, R. M. I. Kapsa, Biomater. Sci. 1 (2013) 983-995.

[6] D.-F. Li, H.-J. Wang, J.-X. Fu, W. Wang, X.-S. Jia, J.-Y. Wang, The Journal of Physical Chemistry B 112 (2008) $16290-16299$.

[7] R. B. Pernites, R. R. Ponnapati, R. C. Advincula, Advanced Materials 23 (2011) 3207-3213.

[8] R. S. Bhatta, Y. Y. Yimer, D. S. Perry, M. Tsige, The Journal of Physical Chemistry B 117 (2013) 10035 - 10045.

[9] H. J. C. Berendsen, J. R. Grigera, T. P. Straatsma, The Journal of Physical Chemistry 91 (1987) 6269 - 6271.

[10] J.-P. Ryckaert, G. Ciccotti, H. J. Berendsen, Journal of Computational Physics 23 (1977) 327 - 341.

[11] G. Scocchi, D. Sergi, C. D’Angelo, A. Ortona, Phys. Rev. E 84 (2011) 061602.

[12] C. M. Tenney, R. T. Cygan, Environmental Science \& Technology 48 (2014) 2035-2042.

[13] D. Vanzo, D. Bratko, A. Luzar, The Journal of Chemical Physics 137 (2012) 034707.

[14] S. Plimpton, Journal of Computational Physics 117 (1995) 1 - 19.

[15] R. Hockney, J. Eastwood, Computer Simulation Using PARTICLEs, Adam Hilger, New York, 1988.

[16] Y. Y. Yimer, A. Dhinojwala, M. Tsige, The Journal of Chemical Physics 137 (2012) 044703.

[17] Y. Y. Yimer, M. Tsige, The Journal of Chemical Physics 137 (2012) 204701.

[18] T. J. Kramer, A. R. Chew, T. Schiros, I. Kymissis, I. P. Herman, Journal of Vacuum Science \& Technology B 31 (2013) 031209.

[19] L. Robinson, J. Isaksson, N. D. Robinson, M. Berggren, Surface Science 600 (2006) L148 - L152.

[20] Z. Wu, T. Song, Y. Jin, B. Sun, Applied Physics Letters 99 (2011) 143306.

[21] R. Kokubu, Y. Yang, Phys. Chem. Chem. Phys. 14 (2012) 8313 - 8318

[22] T. Young, Phil. Trans. R. Soc. 95 (1805) 6587.

[23] C. Vegaa, E. de Miguel, The Journal of Chemical Physics 126 (2007) 154707. 


\section{Graphical Abstract}

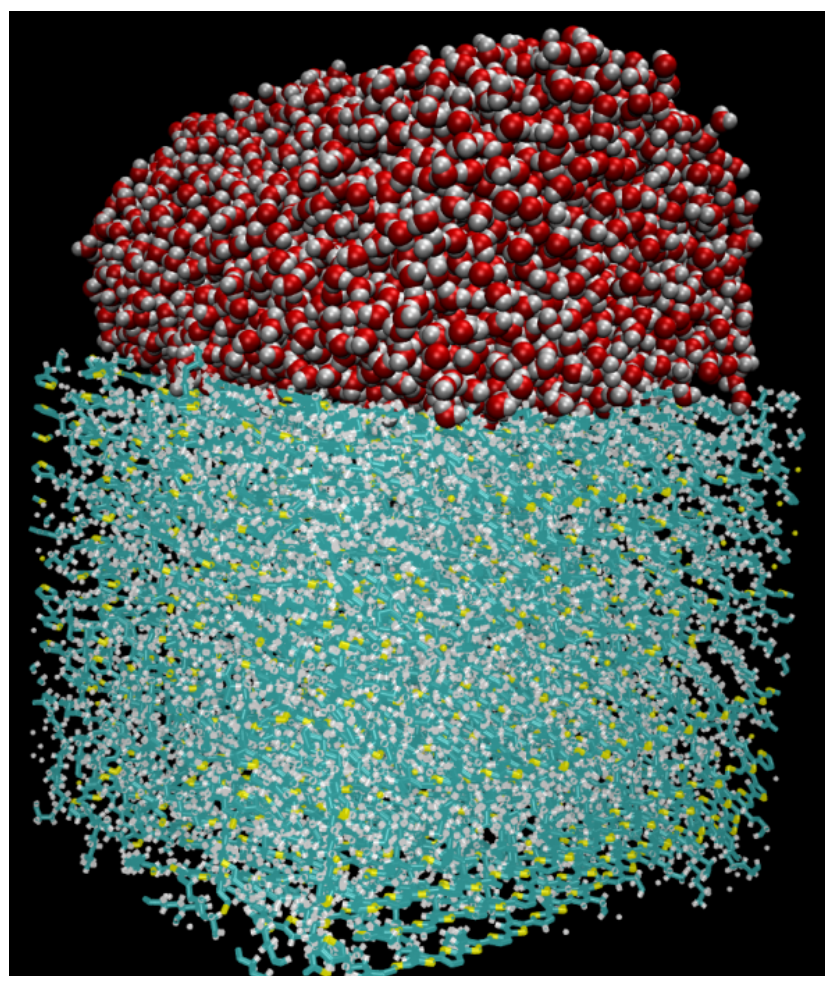

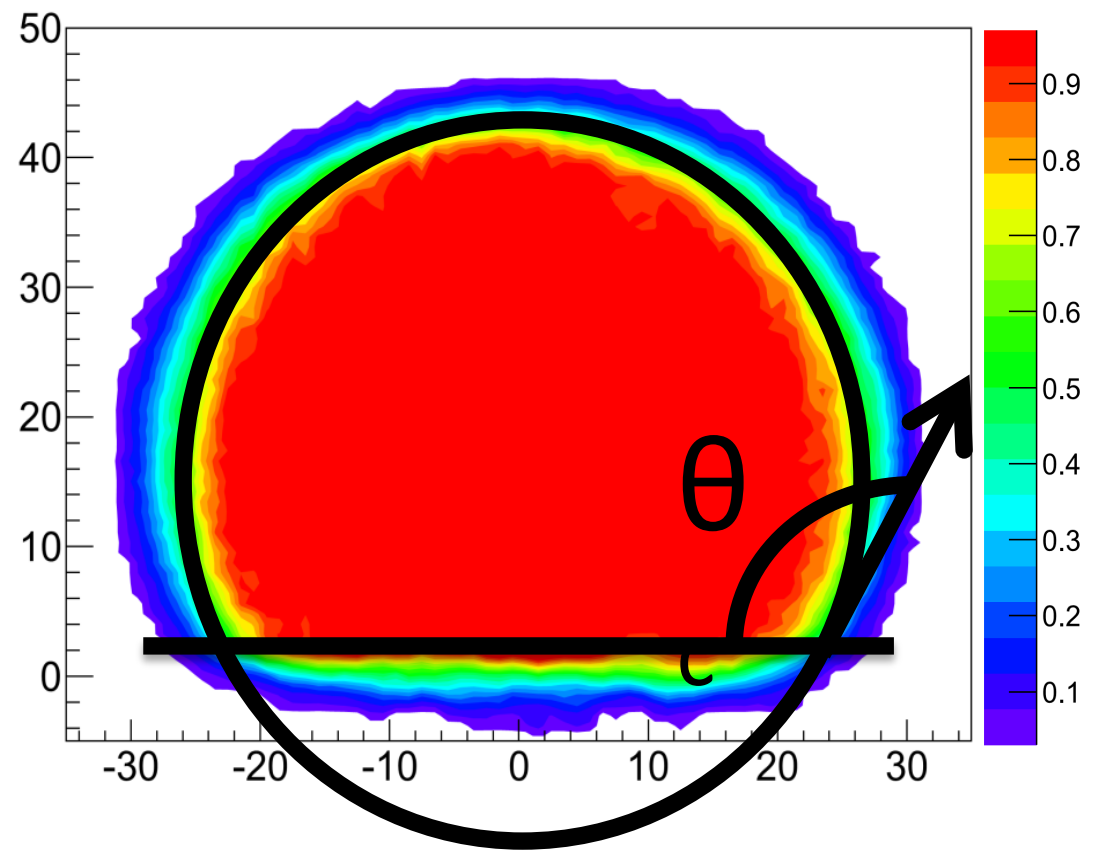

